\title{
Novel Assistive Device for Tomographic Ultrasound Neck Im- aging vs. Freehand
}

\author{
Marcel Köhler, Otto-von-Guericke University, Magdeburg, Germany, marcel.koehler@st.ovgu.de \\ Elmer Jeto Gomes Ataide, Otto-von-Guericke University, Magdeburg, Germany, \\ elmer.gomesataide@ovgu.de \\ Jens Ziegle, Otto-von-Guericke University, Magdeburg, Germany, jens.ziegle@ovgu.de \\ Axel Boese, Otto-von-Guericke University, Magdeburg, Germany, axel.boese@ovgu.de \\ Michael Friebe, Otto-von-Guericke University, Magdeburg, Germany, Michael.friebe@ovgu.de
}

\section{Introduction}

Ultrasound (US) systems that utilize tracking methods to produce 3D volumes are called 3D or tomographic ultrasound systems (tUS). The tUS systems use US systems coupled with tracking systems (electromagnetic(EM), optical, mechanical and hybrid) to reconstruct 3D volumes from 2D US image slices. Initial prototypes developed were tested but did not yield the desired results. Based on those initial results this paper deals with the use of a systematic designing approach to conceptualize and build an improved prototype for assisted 3D US acquisitions.

\section{Methods}

The new prototype was designed based on the guidelines specified by VDI 2221 by the Association of German Engineers. This designing approach starts with a systematic task analysis to generate a comprehensive list of requirements and boundary conditions for the design. The most important requirements were that the patient's head / neck must be stable during the scanning process, the circulation radius of the US probe needs to be adaptable to fit different neck sizes, the design must not include any magnetic parts materials because of their interference with the electromagnetic tracking system and the device needs to be put on / taken off in 5 steps or less and without use of additional tools.

\section{Results and Conclusion}

The prototype was tested on 5 male subjects. The test setup included two scan acquisition approaches: freehand tUS scans and assisted scans with the prototype. In this work we used an EM tracking system (PIUR Imaging, Vienna, Austria) coupled with an US system (9L probe and Logiq E9, GE Wisconsin, USA). Experiments from both scanning methods (freehand and assisted) generate measurable results. The difference lies in the reconstruction and subsequent visualization of the $3 \mathrm{D}$ volumes. Volume reconstruction and visualization from freehand scans appear more to have more irregularities and deformations. Assisted volumes depict a more uniform reconstruction and visualization. This is due to the fact that the probe always follows a 'standard' guided path. If this acquisition method and prototype can be further developed it could be also used for assessing other body parts like internal structures in the abdominal region. 


\title{
Impact of artificial airway resistances on regional ventilation distribution during airway closure
}

\author{
Melanie März, Institute of Technical Medicine, Furtwangen University, Jakob-Kienzle-Straße 17, Villingen- \\ Schwenningen, Germany, maer@hs-furtwangen.de \\ Sarah Howe, Centre for Bio-Engineering, University of Canterbury, Christchurch, New Zealand, \\ sarah.howe@pg.canterbury.ac.nz \\ Bernhard Laufer, Institute of Technical Medicine, Furtwangen University, Villingen-Schwenningen, Germany, \\ lab@hs-furtwangen.de \\ Knut Moeller, Institute of Technical Medicine, Furtwangen University, Villingen-Schwenningen, Germany, \\ moe@hs-furtwangen.de \\ Sabine Krueger-Ziolek, Institute of Technical Medicine, Furtwangen University, Villingen-Schwenningen, \\ Germany, krue@hs-furtwangen.de
}

\section{Introduction}

Electrical impedance tomography (EIT), a non-invasive and radiation-free imaging technique can be used in pulmonary function monitoring for determining regional ventilation distribution within the lung. Gold standard in pulmonary function monitoring is spirometry/body plethys-mography, a method using forced breathing maneuvers to obtain global lung function parameters. However, this method is heavily dependent on the cooperation of the patients.

\section{Methods}

Within this observational study, a method under normal breathing was tested with 5 healthy volunteers, which provides regional information about ventilation distribution. The occlusion method Rocc, a method for determining airway resistance, was used to create a short-term airway closure. Regional ventilation during the airway closure was examined with EIT. Simultaneously four different artificial airway resistances were used to simulate airway obstructions.

\section{Results}

Results show that EIT in combination with the ROcc method is suitable for the detection of regional differences in ventilation during airway closure for all four artificial airway resistances. Although the sum of relative impedances at the end of the shutter maneuver are smaller (nearly $-0.100 \mathrm{AU}$ ) for the airway resistances $\varnothing$ $12.5 \mathrm{~mm}, \varnothing 10.5 \mathrm{~mm}$ and $\varnothing 9.5 \mathrm{~mm}$ than for the smallest one with $\varnothing 30.0 \mathrm{~mm}(\sim-0.070 \mathrm{AU})$, the changes in impedance from the start to the end of the shutter maneuver differs only slightly between the four artificial airway resistances. All impedance changes are in the range of 0.100 to $0.130 \mathrm{AU}$.

\section{Conclusion}

The combination of EIT and the ROcc method provides not only global parameters such as airway resistance under normal breathing conditions, but also results of regional ventilation, which could enable the identification of areas affected by airway obstructions. However, the obtained results indicate that EIT might be a useful tool in the diagnosis and follow-up of obstructive lung diseases. 


\section{Global Inhomogeneity Index Evaluation of a DCT-based EIT Lung Imaging}

Rongqing Chen, Institute of Technical Medicine, Furtwangen University, Jakob-Kienzle-Str. 17, VSSchwenningen, Germany. e-mail: chr@fs-furtwangen.de

Knut Möller, Institute of Technical Medicine, Furtwangen University, Jakob-Kienzle-Str. 17, VSSchwenningen, Germany. e-mail: moe@fs-furtwangen.de

\section{Introduction}

Combination of imaging modalities of CT and EIT to roughly restrict EIT image to CT generated anatomy generates a novel DCT-based EIT approach. This approach includes detailed prior information about both the thorax contour and lung shape obtained from the discrete cosine transformation (DCT) of the CT image, which as a side effect is resulting in improved interpretability for clinicians. The objective of this work is to evaluate the plausibility of a novel DCT-based EIT lung imaging method against the traditional GaussNewton one step method in clinical settings. In a first step Global Inhomogeneity (GI) index is used for comparison, which indicates the difference of the volume distribution within a ventilation period.

\section{Methods}

Taken retrospectively from a former study, EIT data was evaluated using both reconstruction methods. For different phases of ventilation, EIT images are analyzed with respect to the global inhomogeneity $(\mathrm{GI})$ index for comparison.

\section{Results}

A significant less variant $\mathrm{Gl}$ index was observed in the DCT-based method, compared to the index from classical method.

\section{Conclusion}

The DCT-based method generates more accurate lung contour yet decreasing the essential information in the image which affects the GI index. These preliminary results must be consolidated with more patient data in different breathing states. 


\title{
A Deep-Learning Approach to Detect Fiducials in Planar X-Ray Images for Undistortion of Conventional C-Arm Images
}

\author{
Alvarez-Gomez Julio*, julio.agomez@uni-siegen.de \\ Spieß Arne*, arne.spiess@student.uni-siegen.de \\ Prof. Dr.-Ing. Roth Hubert*, hubert.roth@uni-siegen.de \\ Dr.-Ing. Wahrburg Jürgen*, wahrburg@zess.uni-siegen.de \\ ${ }^{*}$ Center for Sensor Systems (ZESS), Univerisity of Siegen, Siegen, Germany
}

\section{Introduction}

In some applications such as 2D-3D registration, undistorted images are required to achieve optimal results. These types of images can be obtained from a distortion-free C-arm (flat-panel detector) or by undistorting the images given from a conventional C-arm (analog image intensifier.) Undistorting images require a plate with fiducials connected to the C-arm detector. Detecting fiducials is affected by differences in the image contrast due to elements in the background. Therefore, the results vary from image to image and could require manual tuning of parameters. We propose a deep-learning approach for detecting undistortion-platefiducials in X-ray images to overcome the drawbacks previously stated.

\section{Methods}

We created an undistortion plate of 60 fiducials in a grid of $8 \times 8$ without corners. We used a Ziehm Vision Carm and took 1120 X-Rays in different poses of the C-arm. Every X-ray was afterward cut into 60 subimages of $32 \times 32$ pixels, and each of the sub-images was labeled with the $X$ and $Y$ coordinate of the fiducial position. These 67200 sub-images were used for training a convolutional neural network (CNN). The topology used consisted of three layers of convolutions plus max-polling and, in the end, a fully connected neural network with three hidden layers.

\section{Results}

Comparing the CNN and a traditional image processing method based on the Hough Circle algorithm, we found that the detected fiducials using the traditional method give a similar fiducial positioning error. Nevertheless, the fiducial detection rate goes from $89.7 \%$ using the traditional method to $100 \%$ with the developed CNN.

\section{Conclusion}

We propose to detect undistortion plate finducials in planar X-Ray images coming from conventional C-Arms using a Deep-Learning approach. The results show that the detection rate and precision of our deep-learning approach guarantee the undistortion of conventional C-Arm images.

Acknowledgment: Part of this work is funded by the German Federal Ministry of Education and Research (KMU-innovativ: contract number 13GW0175B) 


\section{Deep-learning based reconstruction of the stomach from monoscopic video data}

Ralf Hackner, Fraunhofer Institute for Integrated Circuits IIS, Erlangen, Germany, ralf.hackner@iis.fraunho$\underline{\text { fer.de }}$

Martin Raithel, Malteser Waldkrankenhaus St. Marien, Erlangen, Germany, Martin.Raithel@waldkrankenhaus.de

Edgar Lehmann, Fa. E\& L Medical Systems, Erlangen, Germany, edgar.lehmann@eundl.de

Thomas Wittenberg, FraunhoferInstitute for Integrated Circuits IIS, Erlangen, Germany, thomas.wittenberg@iis.fraunhofer.de.

\section{Introduction}

For the gastroscopic examination of the stomach, the restricted field of view related to the „keyhole“ perspective of the endoscope is known to be a visual limitation. Thus, a panoramic extension can enlarge the field of vision, supports the endoscopist during the examination, and ensures that all of the inner stomach walls are visually inspected.

\section{Methods}

To compute such a panorama of the stomach, knowledge about the geometry of the underlying structure is required. Structure from motion one an approach to reconstruct the necessary information about the 3D-structure from monocular image sequences as provided by a gastroscope. We examine and evaluate an existing deep neuronal network (DeMoN) for stereo reconstruction, in order to approximate the geometry of stomach parts from a set of consecutive acquired image pairs from gastroscopic videos.

\section{Results}

We extracted 48 image pairs from gastroscopic video streams of the stomach which were reconstructed using the DeMoN network. Evaluation was done qualititative. Additionally we manually 3D-scanned a silicon stomach phantom and compared the expected shapes with the reconstructed shapes

\section{Conclusion}

We were able to yield 3D- approximations of stomach parts based on pairs of monocular gastroscopic images. The quality of the obtained depth maps is sufficient for a subsequent generation of 3D-panoramas in most of our test cases, even though the DeMoN network was originally trained with datasets from a different domain. Only small motions of the gastroscope in a short time interval are were necessary to extract sufficient information for a 3D-reconstruction. 